\title{
The added value of longitudinal black-blood cardiovascular magnetic resonance angiography in the cross sectional identification of carotid atherosclerotic ulceration
}

\author{
Wei Yu ${ }^{1,2}$, Hunter R Underhill ${ }^{1}$, Marina S Ferguson ${ }^{1}$, Daniel S Hippe ${ }^{1}$, \\ Thomas S Hatsukami ${ }^{3}$, Chun Yuan ${ }^{1}$ and Baocheng Chu*1
}

Address: ${ }^{1}$ Department of Radiology, University of Washington, Seattle, WA, USA, ${ }^{2}$ Department of Radiology, Beijing Anzhen Hospital, Capital Medical University, Beijing, PR China and '3Department of Surgery, Vascular Surgery Division, University of Washington, Seattle, WA, USA

Email: Wei Yu - yuwei02@gmail.com; Hunter R Underhill - hunterru@u.washington.edu; Marina S Ferguson - msf2@u.washington.edu; Daniel S Hippe - dhippe@u.washington.edu; Thomas S Hatsukami - tomhat@u.washington.edu; Chun Yuan - cyuan@u.washington.edu; Baocheng Chu* - chubc@u.washington.edu

* Corresponding author

Published: 18 August 2009

Journal of Cardiovascular Magnetic Resonance 2009, I I:3 I doi:I0.1 I86/I532-429X-I I-3 I

This article is available from: http://www.jcmr-online.com/content/I I/I/3I

(c) 2009 Yu et al; licensee BioMed Central Ltd.

This is an Open Access article distributed under the terms of the Creative Commons Attribution License (http://creativecommons.org/licenses/by/2.0), which permits unrestricted use, distribution, and reproduction in any medium, provided the original work is properly cited.
Received: 27 March 2009

Accepted: 18 August 2009

\begin{abstract}
Background: Carotid atherosclerotic ulceration is a significant source of stroke. This study evaluates the efficacy of adding longitudinal black-blood (BB) cardiovascular magnetic resonance (CMR) angiography to cross-sectional CMR images in the identification of carotid atherosclerotic ulceration.

Methods: Thirty-two subjects ( 30 males and two females with ages between 48 and 83 years) scheduled for carotid endarterectomy were imaged on a I.5T GE Signa scanner using multisequence [3D time-of-flight, TI, proton density, T2, contrast enhanced TI], cross-sectional CMR images and longitudinal BB CMR angiography $(0.625 \times 0.625 \mathrm{~mm} /$ pixel). Two rounds of review (round I: cross-sectional CMR images alone and round 2: cross-sectional CMR images plus longitudinal BB CMR angiography) were conducted for the presence and volume measurements of ulceration. Ulceration was defined as a distinct depression into the plaque containing blood flow signal on cross-sectional CMR and longitudinal BB CMR angiography.

Results: Of the 32 plaques examined by histology, 17 contained 21 ulcers. Using the longitudinal BB CMR angiography sequence in addition to the cross-sectional CMR images in round 2 , the sensitivity improved to $80 \%$ for ulcers of at least $6 \mathrm{~mm}^{3}$ in volume by histology and $52.4 \%$ for all ulcers, compared to $30 \%$ and $23.8 \%$ in round I, respectively. There was a slight decline in specificity from $88.2 \%$ to $82.3 \%$, though both the positive and negative predictive values increased modestly from $71.4 \%$ to $78.6 \%$ and from $48.4 \%$ to $58.3 \%$, respectively.
\end{abstract}

Conclusion: The addition of longitudinal BB CMR angiography to multisequence cross-sectional CMR images increases accuracy in the identification of carotid atherosclerotic ulceration. 


\section{Background}

Stroke is the third most common cause of death and a leading cause of serious, long-term disability worldwide [1]. Extracranial carotid disease accounts for $20-30 \%$ of such ischemic events [2]. Carotid atherosclerotic ulceration is a significant contributing source of cerebral ischemic incidents or stroke, along with other plaque features such as intraplaque hemorrhage and thrombosis [3$10]$.

Although angiography, specifically digital subtraction angiography (DSA), time-of-flight cardiovascular magnetic resonance (CMR) angiography, or computed tomography angiography (CTA), has become the standard approach to carotid artery disease assessment, these modalities are limited by their inability to show the outer wall of the vessel. Black-blood CMR can depict the actual plaque size by imaging both the lumen and the outer wall of an artery. Studies comparing in vivo black-blood CMR with corresponding endarterectomy specimens confirm the ability of black-blood CMR to accurately visualize atheroma size [11-13]. On the other hand, time-of-flight CMR angiography yields lower estimates of luminal narrowing compared to black-blood CMR angiography $[13,14]$, and underestimates the presence of high-risk plaque features [15]. These findings highlight the need for an improved clinical vessel wall imaging method to accurately grade the severity and stage of carotid atherosclerosis [15].

Previous studies have shown good sensitivity and specificity in the identification of carotid atherosclerotic plaque components and the condition of the fibrous cap overlying the necrotic core using multisequence high-spatialresolution cross-sectional CMR imaging [11,16-20]. Identification of fibrous cap rupture is highly associated with recent transient ischemic attack or stroke [17]. Furthermore, studies have demonstrated that detection of carotid atherosclerotic ulceration is possible using these multisequence cross-sectional CMR images $[21,22]$. However, cross-sectional CMR images may suffer from partial volume effects due to the intrinsic morphological changes of the carotid bulb [23-25]. Flow artifacts that mimic plaques [23] and may potentially confound the clear detection of ulcerations occur frequently in this region. The addition of longitudinal black-blood (BB) CMR angiography may reduce errors in classification due to partial volume effects by producing images with greater resolution in the long-axis view of the carotid artery. Longitudinal BB CMR angiography is therefore advocated for preoperative assessment of the long-axis extent of advanced lesions and for determining the precise location of the carotid bifurcation [26].
In this study, we hypothesize that adding the longitudinal BB CMR angiography to multisequence high-spatial-resolution cross-sectional CMR images can improve the ability of CMR in the characterization of carotid atherosclerotic ulceration.

\section{Materials and methods Subjects}

From 1998 to 2004, 58 patients (50 males and 8 females, ages between 48 and 83, and median age 69 years) scheduled for carotid endarterectomy at the University of Washington Medical Center or VA Puget Sound Health Care System underwent preoperative carotid CMR after obtaining informed consent. All subjects either experienced a transient ischemic attack, amaurosis fugax, or stroke within 6 months of their surgery and had $>50 \%$ carotid stenosis by duplex ultrasound or were asymptomatic with $>80 \%$ carotid stenosis.

Twenty-two subjects were excluded from this study because there was no BB CMR angiography available, three subjects were excluded due to poor image quality [20], and one subject was excluded because the histology specimen was too damaged to review. Of the original 58 subjects, 32 (30 males and two females with ages 48-83) with both BB CMR angiography and histology of their excised plaque were analyzed.

\section{CMR acquisition}

The carotid CMR scans were performed within one week prior to carotid endarterectomy. Patients were imaged with a 1.5T GE Signa scanner (Horizon EchoSpeed, General Electric Healthcare, Milwaukee, USA) using a custom designed 4-element, phased-array carotid surface coil to improve the signal-to-noise performance [27].

The carotid CMR scans were performed using a standard imaging protocol, which includes longitudinal BB CMR angiography and five contrast weighted cross-sectional images. The longitudinal BB CMR angiography (Figure 1) was acquired centered on the carotid bifurcation identified by a 2-dimensional time-of-flight (2D TOF) on the operative side. The scan used a multi-slice double inversion recovery 2D fast spin echo (FSE) sequence [repetition time (TR), $1800 \mathrm{~ms}$; effective echo time (TE), $9.5 \mathrm{~ms}$; field of view (FOV), $160 \times 160 \mathrm{~mm}$; matrix, $256 \times 256$ (inplane resolution: $0.625 \times 0.625 \mathrm{~mm} /$ pixel); thickness, 2 $\mathrm{mm}$; Section gap, $0 \mathrm{~mm}$; and number of excitation (NEX), 2]. A total of six images were obtained per carotid artery [26].

Five contrast weighted cross-sectional images [3-dimensional time-of-flight (3D TOF), proton density (PD), T2-, and pre- and post-contrast enhanced T1-weighted images] of the carotid arteries were acquired using the carotid 


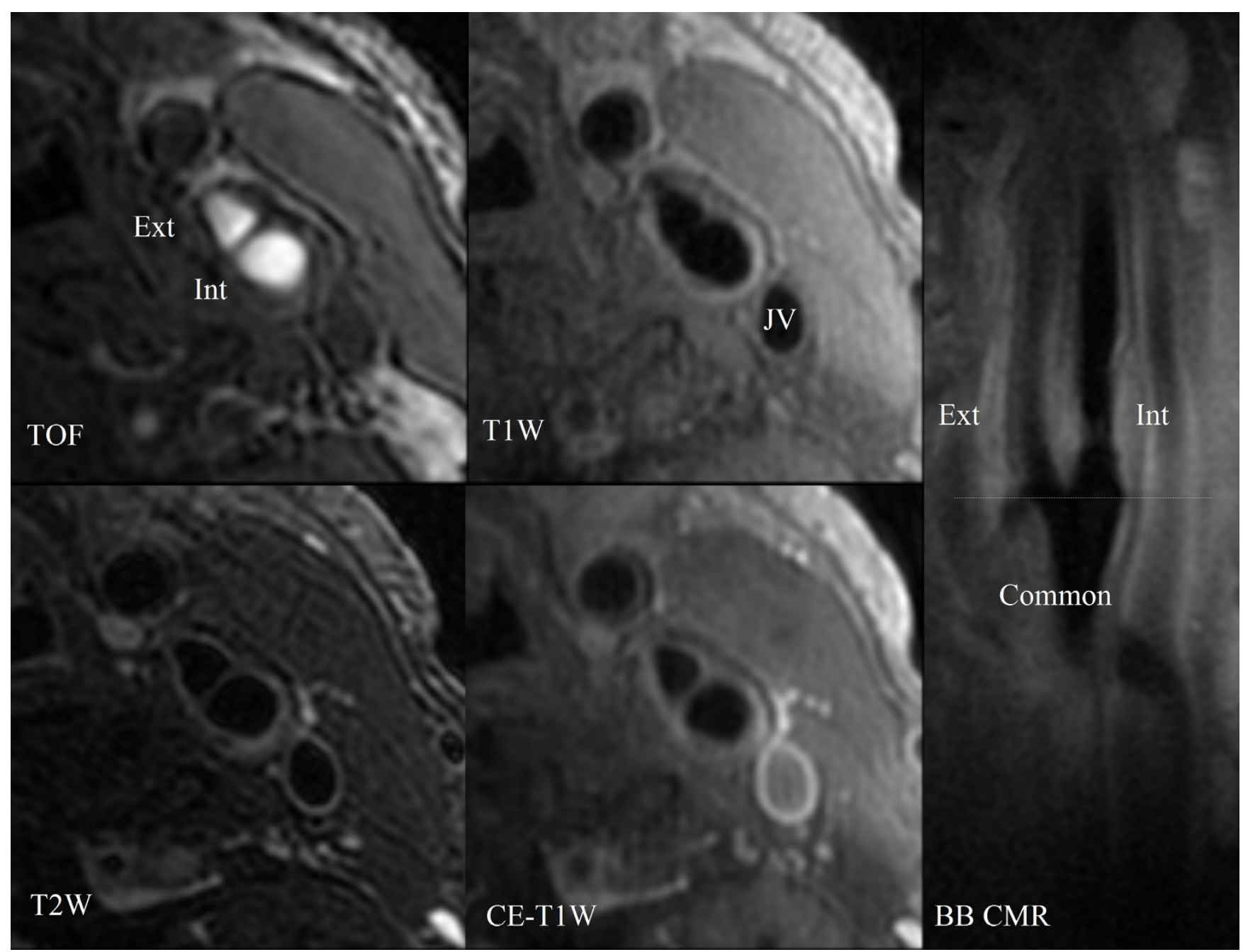

Figure I

Example of carotid multisequence cross-sectional CMR images and a longitudinal black-blood CMR (BB CMR) angiography. Four contrast-weighted CMR images [3-dimensional time-of-flight (TOF), TI-weighted (TIW), T2-weighted (T2W), and contrast-enhanced TI-weighted (CE-TIW)] are matched using the carotid bifurcation (dashed line) seen on the longitudinal BB CMR angiography as the landmark. Proton density weighted image is not shown. Int = internal carotid artery. Ext $=$ external carotid artery. Common $=$ common carotid artery. $\mathrm{JV}=$ jugular vein.

bifurcation as a landmark. The imaging protocol consisted of the following sequences: 1) 2D pre-contrast quadruple inversion recovery (QIR) T1-weighted FSE (TR, $800 \mathrm{~ms}$; TI 1/TI 2, 375/125 ms; effective TE, $11 \mathrm{~ms}$ ) [28]; 2) 2D cardiac gated PD weighted FSE (TR, 3 R-R intervals; effective TE, $17.9 \mathrm{~ms})$; 3) 2D cardiac gated T2-weighted FSE (TR, 3 R-R intervals; effective TE, $68 \mathrm{~ms}$ ) [29]; 4) 3D TOF CMR angiography (TR, $23 \mathrm{~ms}$; TE, $3.6 \mathrm{~ms}$; flip angle, 25 degrees); and 5) 2D post-contrast QIR T1-weighted FSE with the same parameters as for the pre-contrast T1weighted FSE sequence. A gadolinium-based contrast agent (Omniscan, GE Healthcare) was administered intravenously at a dose of $0.1 \mathrm{mmol} / \mathrm{kg}$ body weight for postcontrast QIR T1-weighted FSE sequence (Figure 1).
All cross-sectional images were obtained with a field of view of $160 \times 160 \mathrm{~mm}$, matrix size of $256 \times 256$ (in-plane resolution: $0.625 \times 0.625 \mathrm{~mm} /$ pixel), section thickness of $2 \mathrm{~mm}$, and NEX of 2. Section gap was $0 \mathrm{~mm}$ for pre- and post-contrast enhanced T1-, PD, and T2-weighted images, and $-1 \mathrm{~mm}$ for 3D TOF (1 mm overlapping between adjacent images). The coverage of each carotid artery was 24 $\mathrm{mm}$ (12 images) for pre- and post-contrast enhanced T1-, $36 \mathrm{~mm}$ (18 images) for PD and T2-weighted images, and $40 \mathrm{~mm}$ (40 images) for 3D TOF source images.

\section{CMR matching and review}

The five contrast weighted cross-sectional CMR images were matched using the carotid bifurcation as a landmark. 
CMR images were then analyzed in two separate rounds of review. The interval between the reviews was 103 days. The first round used only the five cross-sectional contrast weighted CMR images. All CMR slices were evaluated for the presence of ulcer by one reviewer (W.Y.) and were peer reviewed by another reviewer (H.U.). Ulcer was defined as a surface disruption with a depression into the plaque containing a disorganized blood flow signal on the five contrast-weighted cross-sectional CMR images. In the second round, $\mathrm{BB}$ CMR angiography series were included and the first round of CMR review was revisited. The reviewer (W.Y.) modified the results of the first round if her interpretation changed with the addition of BB CMR angiography. The second round was also peer reviewed by H.U. In the second round of review, the same ulcer defini- tion was applied to both the cross-sectional slices and BB CMR angiography. If disagreement occurred, consensus opinion was adopted following discussion between the two reviewers. Both reviewers were blinded to histology during the two rounds of review (Figure 2).

During both rounds, the area of each ulcer was measured from every slice on which it was identified using custombuilt software package (CASCADE) [30].

\section{Histology review}

A reviewer (M.S.F; blinded to CMR data) examined all histology sections. A slice was classified as having ulceration if either of the following was true: 1) The luminal surface (endothelial cells) was disrupted and the length along the

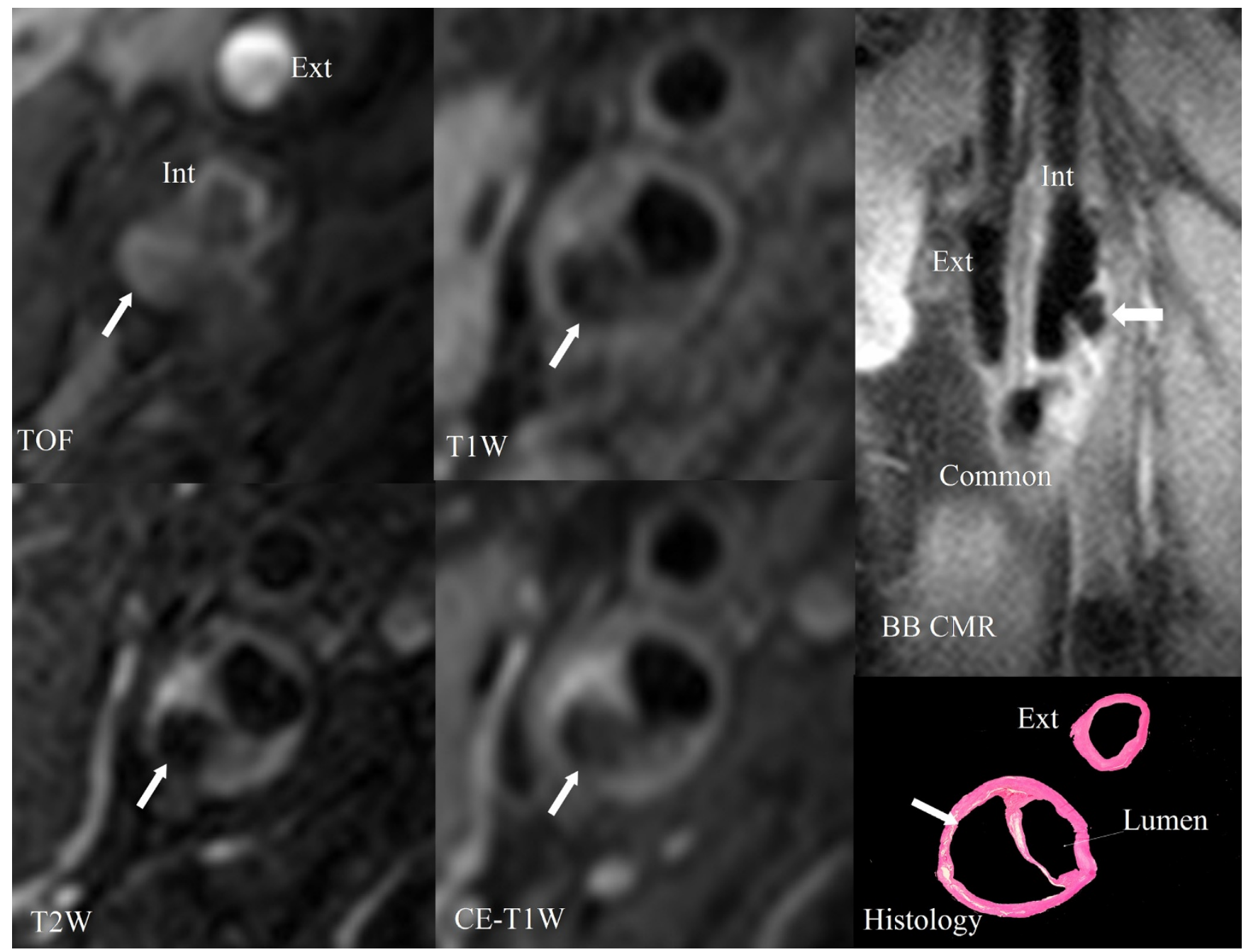

Figure 2

Ulcer identification using multicontrast, cross-sectional CMR images and longitudinal black-blood CMR (BB CMR) angiography. The ulcer (arrows), with a disorganized blood flow signal pattern shown on the cross-sectional CMR images, is well demarcated on the longitudinal BB CMR angiography and confirmed by corresponding histology. Proton density weighted image is not shown. Int $=$ internal carotid artery. Ext $=$ external carotid artery. Common $=$ common carotid artery. 
luminal surface and depth into the wall (both measured on the cross-sections) were each at least $0.5 \mathrm{~mm}$; 2) A disruption on the luminal surface appeared to be a continuation from an adjacent slice already classified as having ulcer, regardless of the length and depth. These criteria were designed to be as close to the CMR criteria as possible while excluding likely processing artifacts, such as shrinkage and distortion of the specimens during processing.

The areas of the disrupted surfaces were measured using the custom software (CASCADE).

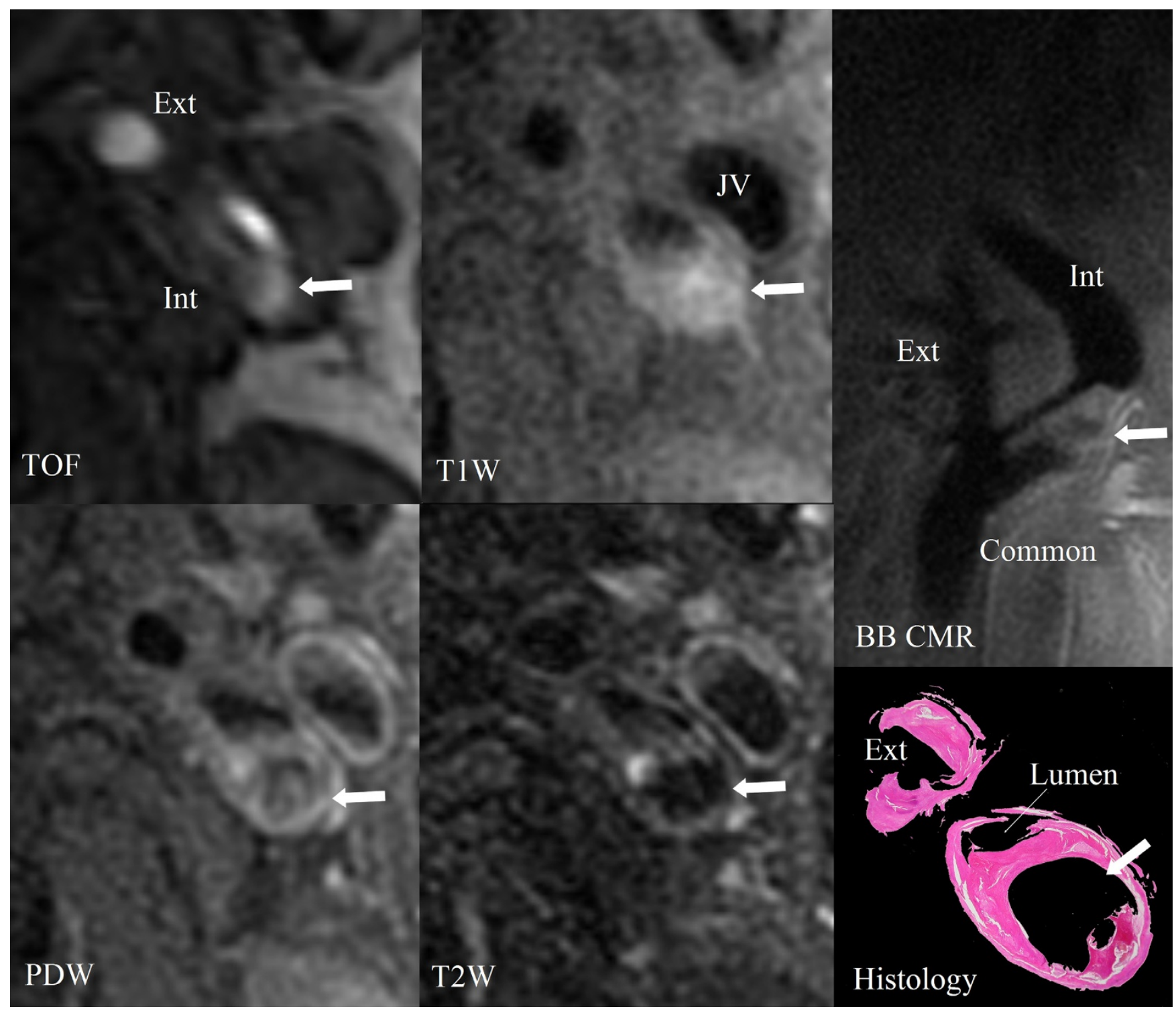

Figure 3

Example of partial volume and flow disturbance in a large penetrating ulcer. The cross-sectional CMR images alone could not verify the presence of the ulcer in the left carotid artery. The signal pattern of the ulcer resembles the signal pattern of intraplaque hemorrhage (arrows). However, the longitudinal black-blood CMR (BB CMR) angiography shows clear separation between the very narrow lumen and the penetrating ulcer due to the improvement of spatial resolution in the longitudinal direction. The flow artifact with this ulcer can be easily identified. Corresponding histology confirms the presence of the ulcer. CE-TIW is not shown. PDW = proton density weighted. Int = internal carotid artery. Ext = external carotid artery. Common = common carotid artery. JV = jugular vein. 


\section{CMR and histology matching}

The contrast weighted cross-sectional CMR images were matched with histology using the carotid bifurcation (Figures 2 and 3) and gross morphological features such as lumen size and shape, wall size and shape, and plaque configuration. At each matched location histology provided the definitive information. In the case where more than one histology section matched a given CMR slice (as histology is collected at every millimeter while CMR images are collected at every 2 millimeters) the final histology ulcer classification was positive if any of the sections were positive.

\section{Statistical analysis}

For both the CMR and histology results, adjacent slices identifying the same ulcer were aggregated into single ulcer observations (the unit of analysis). The volume of each ulcer was computed by multiplying the ulcer area by the distance to the next slice distal.

CMR ulcer classification was evaluated using histology as the gold standard. A true positive was defined as when an ulcer by CMR and an ulcer by histology had at least one location in common. A true negative was defined as when both CMR and histology identified no ulcer in the entire plaque. False positives (negatives) were defined as when an ulcer by CMR (histology) had no locations in common with any ulcers by histology (CMR).

The sensitivity, specificity, positive predictive value and negative predictive value were calculated using the number of true and false positives and negatives of ulcers. As ulcer size was expected to be related to detection performance, sensitivity was plotted versus volume. For each point on the plot, the sensitivity was recalculated using only ulcers greater than the given volume.

The Mann-Whitney rank test was used to assess differences between independent groups of observations, and Spearman's rank correlation was used to assess association between the ulcer volume measurements by CMR and histology. All p-values are two-tailed and all analyses were done using statistical software R2.6.2.

\section{Results}

Of the 32 plaques examined by histology, 17 contained a total of 21 ulcers ( 14 plaques had a single ulcer, 2 had two ulcers, and 1 had three ulcers). After matching with the CMR images, of the 21 ulcers, 8 occurred on only one cross-sectional CMR slice and 9 occurred on three or more slices.

During round 1 (cross sectional review only), 5 true ulcers were detected. Another six ulcers were detected by adding the BB CMR angiography in round 2. The ulcers detected were significantly larger by histology (median $20 \mathrm{~mm}^{3} \mathrm{vs}$. $2.8 \mathrm{~mm}^{3}, \mathrm{p}=0.034$ ).

The Spearman's rank correlation between the volume measurements of the ulcers detected by both CMR and histology in round 2 was $0.60(n=11, p=0.053)$ (Figure 4).

The sensitivity from rounds 1 and 2 were graphed versus ulcer volume by histology, where at each point on the graph, only ulcers of that size or greater were included (Figure 5). In round 2, the sensitivity was $52.4 \%$ for all ulcers $(\mathrm{n}=21)$ and $80.0 \%$ for ulcers at least $6 \mathrm{~mm}^{3}(\mathrm{n}=$ 10). By contrast, the sensitivity from round 1 did not show a clear trend versus size. It was $23.8 \%$ and $30.0 \%$ for all ulcers and ulcers at least $6 \mathrm{~mm}^{3}$, respectively.

Due to an additional false positive, the specificity decreased slightly from $88.2 \%$ in round 1 to $82.3 \%$ in round 2 . However, both the positive and negative predictive values increased modestly in round 2 (from $71.4 \%$ to $78.6 \%$ and from $48.4 \%$ to $58.3 \%$, respectively). When considering only ulcers at least $6 \mathrm{~mm}^{3}$ by histology, the negative predictive value improved from $73.1 \%$ to $90.0 \%$.

In round 2, the true positive ulcers by CMR had larger volumes than the false positives (median $22 \mathrm{~mm}^{3}$ vs. 4.9

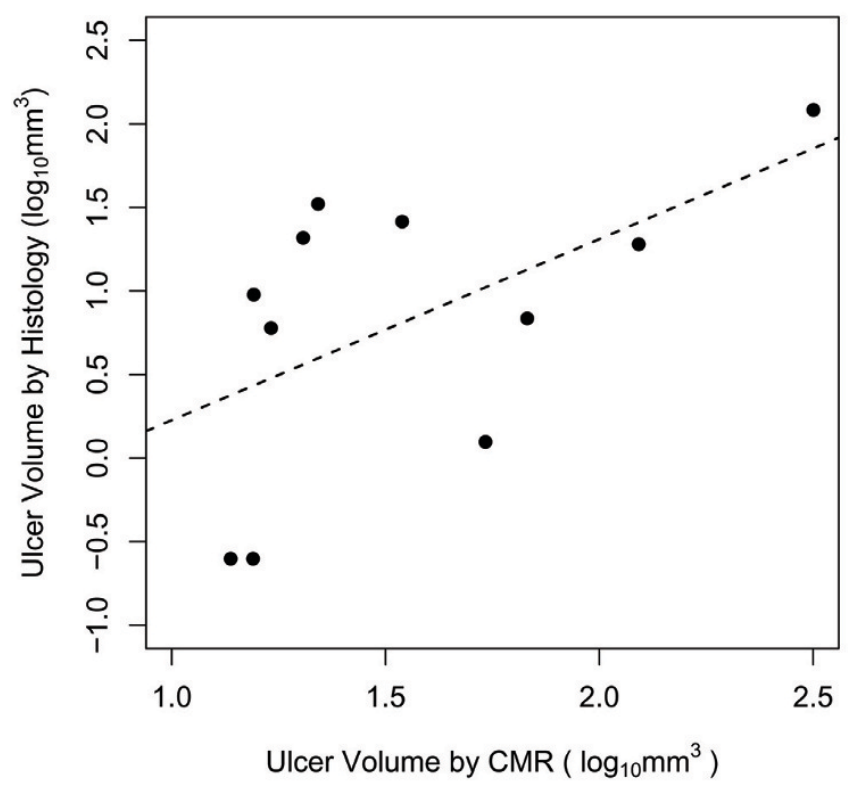

Figure 4

Scatter plots of the ulcer volume on log scale measured by CMR and histology. Only ulcers where histology and CMR agreed in round 2 review (cross-sectional CMR images plus BB CMR angiography) were included $(n=I I)$. Spearmen's rank correlation was calculated as $r=0.60(p=$ 0.053). 


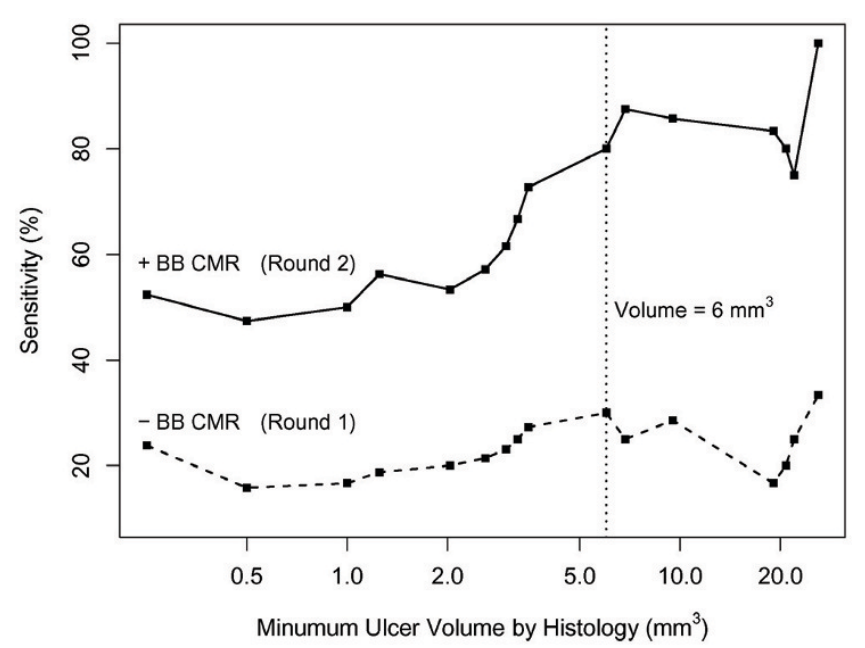

Figure 5

Sensitivity of ulcer identification by CMR with regard to ulcer volume by histology. The sensitivity was calculated at each point in the Figure using only ulcers with the given volume or greater. The sensitivity was uniformly higher in round 2 review (cross-sectional CMR images plus BB CMR angiography), and in particular was notably higher for larger ulcers. Sensitivity did not appear to depend on size in round I review (cross-sectional CMR images alone).

$\mathrm{mm}^{3}, \mathrm{p}=0.005$ ) (Figure 6). In particular, the three false positives were all less than $7 \mathrm{~mm}^{3}$ by CMR whereas the true positives were all greater. $100 \%$ specificity could have been achieved without sacrificing sensitivity simply by discarding those CMR measurements. A similar result, though not significant $(\mathrm{p}=0.19)$, was seen in round 1 .

\section{Discussion}

In this study, the improvement of ulcer identification can be attributed to the combination of cross-sectional imaging, which has greater resolution in the axial plane but two millimeter slice thickness, and longitudinal BB CMR angiography, which provides higher resolution images along the long-axis in the longitudinal direction. This combination improves the ability to identify carotid plaque ulceration by reducing errors in interpretation caused by mis-registration due to partial volume effects (Figure 3) [23-25]. On the other hand, the addition of the longitudinal BB CMR angiography did result in increased false positives. However, since both the positive and negative predictive values increased modestly, higher sensitivity represents improvement by the addition of the longitudinal BB CMR in the ulcer identification.

We also demonstrate that the sensitivity of ulcer detection depends on the size of ulcers. When longitudinal BB CMR angiography was added to the cross-sectional CMR images, the sensitivity was $80 \%$ when only ulcers with vol- ume at least $6 \mathrm{~mm}^{3}$ were evaluated compared to a sensitivity of $52.4 \%$ for all ulcers. There were no ulcers between 3.5 and $6 \mathrm{~mm}^{3}$ (Figure 5) and the overall sample size was small, so the threshold of $6 \mathrm{~mm}^{3}$ could not be estimated precisely. We believe that the dependence of sensitivity on ulcer size is related to the spatial resolution provided by the cross-section CMR images and longitudinal BB CMR angiography. As 3.5-6 $\mathrm{mm}^{3}$ corresponds to an average of 2.4-2.9 pixels in each of the three dimensions of crosssectional CMR images and BB CMR angiography (0.625 $\mathrm{mm}$ /pixel with $2 \mathrm{~mm}$ thickness for each 2D image), our results suggest the minimum requirement of spatial resolution of CMR for consistent ulcer identification is at least 2-3 pixels.

Low sensitivity occurred in the first round of review with cross-sectional CMR images alone. This can be attributed to two factors. First, presence of partial volume effect and/ or flow artifact in the cross-sectional images only led to more false negatives, which subsequently caused lower sensitivity (Figure 3). Second, a conservative approach was used for identifying ulcers by CMR, i.e. ulcers were only identified when the reviewers were very confident of the assessment.

Drawing direct comparisons between the sensitivity and specificity in this study with others is difficult because different imaging modalities and different postprocessing methods were used for ulcer detection [9,31-34]. For ulcer detection, conventional angiography generated a sensitivity of $46 \%$ and a specificity of $74 \%$, as it used limited views (e.g., anterior-posterior/oblique/lateral acquisitions) in the NASCET trial [9]. Single-slice CTA resulted in a sensitivity of $60 \%$ and a specificity of $74 \%$ [31]. Multidetector CTA showed a sensitivity of $94 \%$ and a specificity of $99 \%$ [32]. A combination of axial images, maximum intensity projection, multi-planar reformatting, and volume rendering on Multi-detector CTA resulted in higher sensitivity (94\%) and specificity (99\%) [34]. Therefore, influence of imaging techniques and post processing methods should be taken into account when comparing the accuracy of ulcer detection.

Furthermore, direct comparison between studies of ulceration is difficult because definitions of ulceration vary both among imaging modalities and in histology [9,3133]. In imaging modalities, ulceration is defined by its shape (crater penetrating into a stenotic plaque or double density on "en face" view), its relationship to the vessel lumen (extension of contrast beyond the vascular lumen into surrounding plaque), or irregularity of luminal surface (break in the surface of the plaque with a depth of $\geq 1$ $\mathrm{mm}$ ) [9,31-33]. In histology, ulceration is defined by the integrity of the plaque surface (disruption in the lining of the plaque or a pit and depression in the plaque, fissuring 
- BB CMR (Round 1)

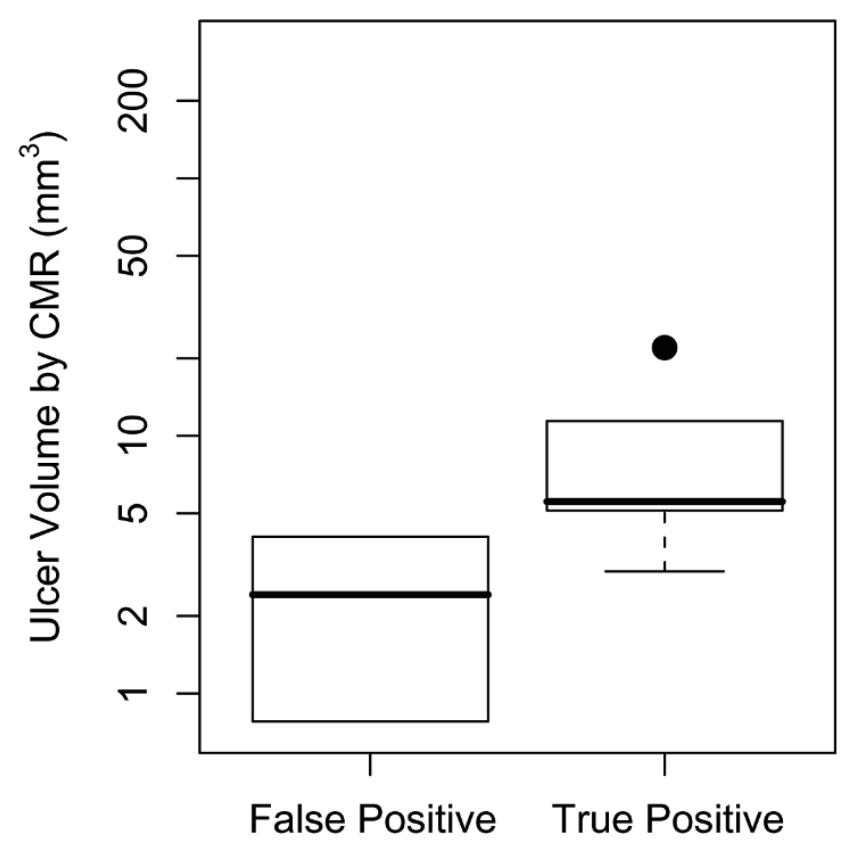

+ BB CMR (Round 2)

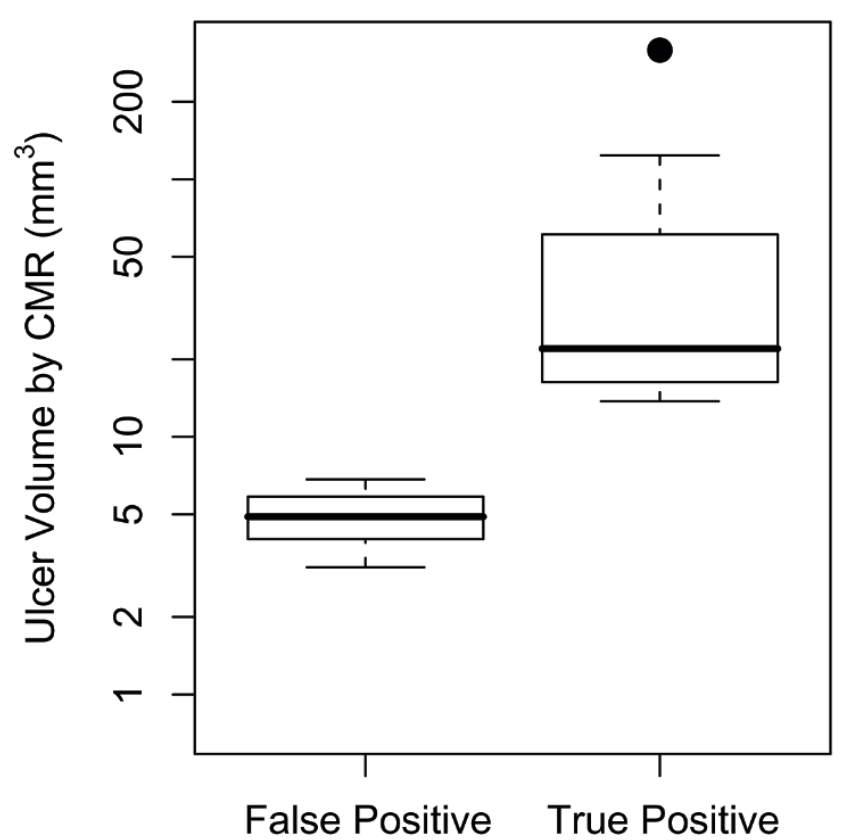

Figure 6

Distributions of CMR ulcer volume measurements, grouped by whether they corresponded to true or false positives. The smaller CMR ulcer volume measurements tended to be false positives, especially in round 2 [P $=0.19$ for round I (cross-sectional CMR images alone, and $p=0.005$ for round 2 (cross-sectional CMR images plus BB CMR angiography).

of the fibrous cap, or break in the surface of the plaque), and/or by penetration depth (approximately $\geq 1 \mathrm{~mm}$ or $\geq 2$ $\mathrm{mm})[9,31-33]$.

In this study, 53\% (17 of 32) plaques examined by histology had ulcers. This is consistent with the prevalence of ulceration reported in other studies [35,36]. Park et al reported that $77 \%$ of carotid lesions removed from symptomatic patients (transient ischemic attack, amaurosis fugax, prior stroke) and $60 \%$ of plaques removed from asymptomatic patients with high-grade stenosis had ulceration [35]. Saam et al reported 25 of 46 (54\%) carotid plaques had fibrous cap rupture [36]. All these patients have similar demographic characteristics as our patients in this study. Therefore, the prevalence of ulceration reported in this study is representative of typical demographic characteristics in a population of subjects who had experienced a transient ischemic attack, amaurosis fugax, or stroke within 6 months of their surgery and had $>50 \%$ carotid stenosis or who were asymptomatic with $>80 \%$ carotid stenosis by duplex ultrasound.

Our study had several limitations. Longitudinal BB CMR angiography is acquired by a $2 \mathrm{D}$ multi-slice double inversion recovery sequence through the centers of internal and external carotid arteries at the carotid bifurcation. If the artery is tortuous, not all segments of the carotid can be covered by this sequence, therefore, ulceration may not be well identified. Implementation of 3D isotropic resolution carotid wall CMR may improve visualization of all segments of carotid artery and allow for better identification of ulceration [37]. In addition, BB CMR angiography may still be insufficient for cases where flow artifact confounds ulcer identification. Use of a motion sensitized driven equilibrium (MSDE) turbo spin echo sequence on 3T CMR may assist in suppression of plaque-mimicking flow artifacts and improve image quality for high-resolution black-blood imaging of carotid arteries [38,39]. This sequence has demonstrated promise in the identification of carotid atherosclerotic ulceration [38]. Furthermore, use of 8-element phased array carotid coils will improve over 4-element coils in the longitudinal coverage and vessel wall signal-to-noise ratio and contrast-to-noise ratio [40]. The number of patients examined is relatively small. Due to the limited number of subjects included, accuracy of adding longitudinal BB CMR angiography needs to be further confirmed by including larger number of subjects in a future study. In addition, due to the small number of subjects, we did not correlate the presence and sizes of ulceration with neurological events in this study. Finally, the study was not blinded. 


\section{Conclusion}

This study demonstrates that adding longitudinal blackblood CMR Angiography to multisequence high-spatialresolution cross-sectional CMR images improves identification of carotid atherosclerotic ulcerations.

\section{Competing interests}

The authors declare that they have no competing interests.

\section{Authors' contributions}

WY performed CMR image review and collection of data. She also contributed to the drafting of the manuscript. HRU performed CMR image peer review. He contributed significantly to the statistical interpretation of data, study design, and revision of the manuscript. MSF was in charge of histological data analysis and revision of the manuscript. DSH collected and analyzed data, performed statistical analysis, prepared figures, and revised the manuscript. CY is a principal investigator of this study's grant. He also assisted in data interpretation and manuscript revision. TSH assisted in data interpretation and was key to revising the critical content of the manuscript. BC participated in CMR image acquisitions, was involved with study design, and was in charge of manuscript preparation and revision of the manuscript. All authors have read and approved submission of this manuscript. The material in the manuscript has not been published and is not being considered for publication elsewhere in whole or in part in any language.

\section{Acknowledgements}

This work was supported by NIH ROIHL56874.

\section{References}

I. Rosamond W, Flegal K, Furie K, Go A, Greenlund K, Haase N, Hailpern SM, Ho M, Howard V, Kissela B, Kittner S, Lloyd-Jones D, McDermott M, Meigs J, Moy C, Nichol G, O'Donnell C, Roger V, Sorlie P, Steinberger J, Thom T, Wilson M, Hong $Y$ : Heart disease and stroke statistics 2008 update: a report from the American Heart Association Statistics Committee and Stroke Statistics Subcommittee. Circulation 2008, I I 7:e25-e 146.

2. Chaer RA, DeRubertis B, Patel S, Lin SC, Kent CK, Faries PL: Current management of extracranial carotid artery disease. Rev Recent Clin Trials 2006, I:293-301.

3. Spagnoli LG, Mauriello A, Sangiorgi G, Fratoni S, Bonanno E, Schwartz RS, Piepgras DG, Pistolese R, Ippoliti A, Holmes DR Jr: Extracranial thrombotically active carotid plaque as a risk factor for ischemic stroke. JAMA 2004, 292: I845-I852.

4. Prabhakaran S, Rundek T, Ramas R, Elkind MS, Paik MC, Boden-Albala $B$, Sacco RL: Carotid plaque surface irregularity predicts ischemic stroke: the northern Manhattan Study. Stroke 2006, 37:2696-270l.

5. Troyer A, Saloner D, Pan XM, Velez P, Rapp JH: Assessment of Carotid Stenosis by Comparison with Endarterectomy Plaque Trial Investigators. Major carotid plaque surface irregularities correlate with neurologic symptoms. J Vasc Surg 2002, 35:74l-747.

6. Fisher M, Paganini-Hill A, Martin A, Cosgrove M, Toole JF, Barnett HJ, Norris J: Carotid plaque pathology: thrombosis, ulceration, and stroke pathogenesis. Stroke 2005, 36:253-257.

7. Redgrave JNE, Lovett JK, Gallagher PJ, Rothwell PW: Histological assessment of 526 symptomatic carotid plaques in relation to the nature and timing of ischemic symptoms: the Oxford plaque study. Circulation 2006, I I 3:2320-2328.
8. Eliasziw M, Streifler JY, Fox AJ, Hachinski VC, Ferguson GG, Barnett $\mathrm{HJ}$ : Significance of plaque ulceration in symptomatic patients with high-grade carotid stenosis. North American Symptomatic Carotid Endarterectomy Trial. Stroke I 994, 25:304-308.

9. Streifler JY, Eliasziw M, Fox AJ, Benavente OR, Hachinski VC, Ferguson GG, Barnett HJ: Angiographic detection of carotid plaque ulceration. Comparison with surgical observations in a multicenter study. North American Symptomatic Carotid Endarterectomy Trial. Stroke 1994, 25: I I30-I I 32.

10. Rothwell PM, Gibson R, Warlow CP: Interrelation between plaque surface morphology and degree of stenosis on carotid angiograms and the risk of ischemic stroke in patients with symptomatic carotid stenosis. On behalf of the European Carotid Surgery Trialists' Collaborative Group. Stroke 2000, 31:615-621.

II. Saam T, Ferguson MS, Yarnykh VL, Takaya N, Xu D, Polissar NL, Hatsukami TS, Yuan C: Quantitative evaluation of carotid plaque composition by in vivo MRI. Arterioscler Thromb Vasc Biol 2005, 25:234-239.

12. Yuan C, Beach KW, Smith LH Jr, Hatsukami TS: Measurement of atherosclerotic carotid plaque size in vivo using high resolution magnetic resonance imaging. Circulation 1998, 98:2666-267I.

13. Babiarz LS, Astor B, Mohamed MA, Wasserman BA: Comparison of gadolinium-enhanced cardiovascular magnetic resonance angiography with high-resolution black-blood cardiovascular magnetic resonance for assessing carotid artery stenosis. J Cardiovasc Magn Reson 2007, 9:63-70.

14. Wasserman BA, Smith WI, Trout III HH, Cannon III RO, Balaban RS, Arai $A E$ : Carotid artery atherosclerosis: In vivo morphologic characterization with gadolinium-enhanced double-oblique MR imaging-Initial results. Radiology 2002, 223:556-573.

I5. Dong L, Underhill HR, Yu W, Ota H, Hatsukami TS, Gao TL, Zhang Z, Oikawa $M$, Zhao X, Yuan C: Geometric and compositional appearance of atheroma in an angiographically normal carotid artery in patients with atherosclerosis. AJNR Am J Neuroradiol 2010 in press.

16. Hatsukami TS, Ross R, Polissar NL, Yuan C: Visualization of fibrous cap thickness and rupture in human atherosclerotic carotid plaque in vivo with high-resolution magnetic resonance imaging. Circulation 2000, I 02:959-964.

17. Yuan C, Zhang SX, Polissar NL, Echelard D, Ortiz G, Davis JW, Ellington E, Ferguson MS, Hatsukami TS: Identification of fibrous cap rupture with magnetic resonance imaging is highly associated with recent transient ischemic attack or stroke. Circulation 2002, 105: |81-185.

18. Sanz J, Fayad Z: Imaging of atherosclerotic cardiovascular disease. Nature 2008, 451 I:953-957.

19. Takaya N, Yuan C, Chu B, Saam T, Underhill H, Cai J, Tran N, Polissar NL, Isaac C, Ferguson MS, Garden GA, Cramer SC, Maravilla KR, Hashimoto B, Hatsukami TS: Association between carotid plaque characteristics and subsequent ischemic cerebrovascular events: a prospective assessment with MRI-initial results. Stroke 2006, 37:818-823.

20. Yuan C, Mitsumori LM, Ferguson MS, Polissar NL, Echelard D, Ortiz G, Small R, Davies JW, Kerwin WS, Hatsukami TS: In vivo accuracy of multispectral magnetic resonance imaging for identifying lipid-rich necrotic cores and intraplaque hemorrhage in advanced human carotid plaques. Circulation 2001, I 04:205 I-2056.

21. Chu B, Yuan C, Takaya N, Shewchuk JR, Clowes AW, Hatsukami TS: Images in cardiovascular medicine. Serial high-spatial-resolution, multisequence magnetic resonance imaging studies identify fibrous cap rupture and penetrating ulcer into carotid atherosclerotic plaque. Circulation 2006, I I 3:e660-e66 I.

22. Chu B, Ferguson MS, Underhill H, Takaya N, Cai J, Kliot M, Yuan C, Hatsukami TS: Images in cardiovascular medicine. Detection of carotid atherosclerotic plaque ulceration, calcification, and thrombosis by multicontrast weighted magnetic resonance imaging. Circulation 2005, I I 2:e3-e4

23. Steinman $D A$, Rutt $B K$ : On the nature and reduction of plaquemimicking flow artifacts in black-blood MRI of the carotid bifurcation. Magn Reson Med 1998, 39:635-64I.

24. Thomas JB, Jong L, Spence JD, Wasserman BA, Rutt BK, Steinman DA: Anthropometric data for magnetic resonance imaging of the carotid bifurcation. J Magn Reson Imaging 2005, 2 I:845-849. 
25. Thomas JB, Aniga L, Che SL, Milner JS, Steinman DA, Spence JD, Rutt BK, Steinman DA: Variation in the carotid bifurcation geometry of young versus older adults: implications for geometric risk of atherosclerosis. Stroke 2005, 36:2450-2456.

26. Yarnykh VL, Yuan C: High-resolution multi-contrast MRI of carotid artery wall for plaque evaluation. In Current protocols in magnetic resonance imaging Edited by: Haacke EM, Lin W. New York: Wiley; 2004:AI.4.I-A I.4.18.

27. Hayes CE, Mathis CM, Yuan C: Surface coil phased arrays for high-resolution imaging of the carotid arteries. J Magn Reson Imaging 1996, 6:109-II2.

28. Yarnykh VL, Yuan C: TI-insensitive flow suppression using quadruple inversion-recovery. Magn Reson Med 2002, 48:899-905.

29. Yarnykh VL, Yuan C: Multislice double inversion-recovery black-blood imaging with simultaneous slice reinversion. J Magn Reson Imaging 2003, 17:478-483.

30. Kerwin W, Xu D, Liu F, Saam T, Underhill H, Takaya N, Chu B, Hatsukami TS, Yuan C: Magnetic resonance imaging of carotid atherosclerosis: plaque analysis. Top Magn Reson Imaging 2007, 18:37|-378.

31. Walker LJ, Ismail A, McMeekin W, Lambert D, Mendelow AD, Birchall $D$ : Computed tomography angiography for the evaluation of carotid atherosclerotic plaque: correlation with histopathology of endarterectomy specimens. Stroke 2002, 33:977-981.

32. Saba L, Caddeo G, Sanfilippo R, Montisci R, Mallarini G: CT and ultrasound in the study of ulcerated carotid plaque compared with surgical results: potentialities and advantages of multidetector row CT angiography. AJNR Am J Neuroradiol 2007, 28:106I-1066.

33. Wintermark M, Jawadi SS, Rapp JH, Tihan T, Tong E, Glidden DV, Abedin S, Schaeffer S, Acevedo-Bolton G, Boudignon B, Orwoll B, Pan $X$, Saloner $D$ : High-resolution $C T$ imaging of carotid artery atherosclerotic plaques. AJNR Am / Neuroradiol 2008, 29:875-882.

34. Runck F, Steiner RP, Bautz WA, Lell MM: MR Imaging: Influence of imaging technique and postprocessing on measurement of intenal carotid artery stenosis. AJNR Am J Neuroradiol 2008, 29:1736-1742

35. Park AE, McCarthy WJ, Pearce WH, Matsumura JS, Yao JS: Carotid plaque morphology correlates with presenting symptomatology. J Vasc Surg 1998, 27:872-878.

36. Saam T, Cai J, Ma L, Cai YQ, Ferguson MS, Polissar NL, Hatsukami TS, Yuan C: Comparison of symptomatic and asymptomatic atherosclerotic carotid plaque features with in vivo MR imaging. Radiology 2006, 240:464-472.

37. Koktzoglou I, Chung YC, Carroll TJ, Simonetti OP, Morasch MD, Li $D$ : Three-dimensional black-blood MR imaging of carotid arteries with segmented steady-state free precession: initial experience. Radiology 2007, 243:220-228.

38. Wang J, Yarnykh VL, Hatsukami TS, Chu B, Balu N, Yuan C: Improved suppression of plaque-mimicking artifacts in black-blood carotid atherosclerosis imaging using a multislice motion-sensitized driven-equilibrium (MSDE) turbo spin-echo (TSE) sequence. Magn Reson Med 2007, 58:973-98I.

39. Yarnykh VL, Terashima M, Hayes CE, Shimakawa A, Takaya N, Nguyen PK, Brittain JH, McConnell MV, Yuan C: Multicontrast black-blood MRI of carotid arteries: comparison between I.5 and 3 Tesla magnetic field strength. J Magn Reson Imaging 2006, 23:691-698.

40. Balu N, Yarnykh VL, Scholnick J, Chu B, Yuan C, Hayes C: Improvements in carotid plaque imaging using a new eight-element phased array coil at 3T. J Magn Reson Imaging 2009 in press.

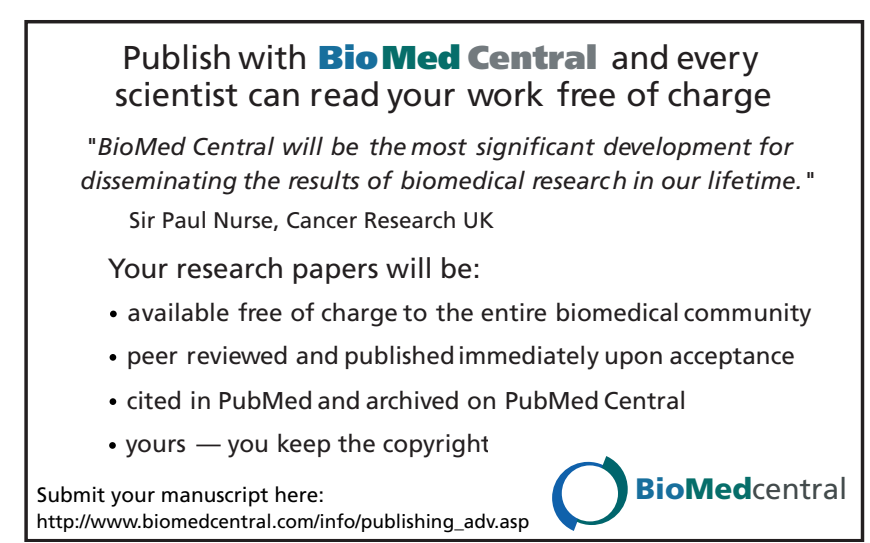

